\title{
Aortic root conservative repair of acute type A aortic dissection involving the aortic root: Fate of the aortic root and aortic valve function
}

Sun Kyun Ro, MD, Joon Bum Kim, MD, Su Kyung Hwang, MD, Sung Ho Jung, MD, Suk Jung Choo, MD, Cheol Hyun Chung, MD, and Jae Won Lee, MD

Objectives: Despite many studies about aortic valve function and aortic root geometry after conservative aortic root repair of acute type A aortic dissection, the results are not always consistent or conclusive. This study aims to evaluate aortic root diameter and aortic valve function after surgery for acute type A aortic dissection involving the aortic root.

Methods: A retrospective review was performed of 196 consecutive patients (age, $56.9 \pm 11.4$ years; 96 men) who underwent conservative aortic root repair including sinotubular junction resuspension for the management of acute type A aortic dissection involving the aortic root.

Results: The 30-day mortality rate was $5.1 \%(n=10)$. During a median follow-up period of $45.3 \pm 36.4$ months, there were 28 deaths and 11 cases of aortic reoperation (proximal reoperation in 1 and distal reoperation in 10). Of the 6-month survivors $(n=177,90.3 \%)$, echocardiography and computed tomography were performed in the late period (>6 months) on $115(65.0 \%)$ and $138(78.0 \%)$, respectively. Significant aortic regurgitation (greater than grade $2+$ ) or root dilatation $(>45 \mathrm{~mm}$ ) was observed in 5 and 19 patients, respectively. Freedom from aortic regurgitation (greater than grade $2+$ ) or root dilatation $(>45 \mathrm{~mm})$ at 5 years was $84.6 \%$ $\pm 3.9 \%$. On the Cox regression analysis, the maximal aortic root diameter at initial presentation was the only significant predictor of aortic regurgitation and aortic root dilatation (hazard ratio, $1.10 ; 95 \%$ confidence interval, $1.02-1.19 ; P=.014)$.

Conclusions: Conservative aortic root repair of acute type A aortic dissection demonstrates acceptable longterm clinical outcomes. However, more aggressive approaches should be considered for patients who have aortic root dilatation because of the risk of developing a root aneurysm after surgery. (J Thorac Cardiovasc Surg 2013; 146:1113-8)

Acute Stanford type A aortic dissection (AD) is one of the most lethal and demanding cardiovascular emergencies. For the surgical management of acute type A AD that involves the aortic root, determining the extent of aortic replacement and choosing the appropriate proximal aortic root procedure are important. To reduce the complexity of the proximal procedures, root conservative repair may be a reasonable option in selected patients because dissection of the proximal portion, management of coronary buttons, and hemostasis at the proximal anastomosis are technically difficult and taxing, especially for surgeons in low-volume centers. $^{1,2}$

\footnotetext{
From the Department of Thoracic and Cardiovascular Surgery, Asan Medical Center, University of Ulsan College of Medicine, Seoul, South Korea.

Disclosures: Authors have nothing to disclose with regard to commercial support.

Received for publication May 16, 2012; revisions received July 18, 2012; accepted for publication Aug 23, 2012; available ahead of print Sept 19, 2012.

Address for reprints: Jae Won Lee, MD, Department of Thoracic and Cardiovascular Surgery, Asan Medical Center, University of Ulsan College of Medicine, 388-1

Pungnap-dong Songpa-gu, Seoul 138-736, South Korea (E-mail: jwlee@amc. seoul.kr).

$0022-5223 / \$ 36.00$

Copyright (C) 2013 by The American Association for Thoracic Surgery

http://dx.doi.org/10.1016/j.jtcvs.2012.08.055
}

Complete replacement of the aortic sinus using root remodeling/reimplantation techniques, on the other hand, may improve the long-term clinical outcomes in terms of the prevention of late aortic complications, such as recurrent dissection, aortic root dilatation, or progression of aortic regurgitation $(\mathrm{AR})^{3,4}$ In this regard, even the late prognosis of conservative aortic root repair, including sinotubular junction resuspension and preservation of the aortic sinuses in the aortic root that are involved in acute type $\mathrm{A} \mathrm{AD}$, is poorly understood, especially in terms of the changes that occur in aortic valve function and aortic root geometry.

The aim of this study was to evaluate the fate of aortic root diameter and aortic valve function after conservative repair of the aortic root in patients with acute type $A \mathrm{AD}$ that involved the aortic root to elucidate the best pathoanatomic substrate that is amenable to a more conservative (rather than a more aggressive) approach in this particular clinical setting.

\section{PATIENTS AND METHODS Study Population}

From January 1999 to July 2011, 289 patients with acute Stanford type A AD underwent emergency surgery at Asan Medical Center, Seoul, South 


\section{Abbreviations and Acronyms \\ $\mathrm{AD}=$ aortic dissection \\ $\mathrm{AR}=$ aortic regurgitation \\ $\mathrm{CI}=$ confidence interval \\ $\mathrm{CT}=$ computed tomography}

Korea. Of these patients, aortic root involvement was diagnosed in 214. The aortic root involvement was defined as $\mathrm{AD}$ extended below the sinotubular junction on the computed tomography (CT) scan. When the sinotubular junction was not clearly demarcated on the CT scan, the horizontal level corresponding to the topmost commissures was determined as the level of sinotubular junction. By excluding patients who had an in situ prosthetic aortic valve $(\mathrm{n}=1)$ and those who underwent concomitant root or aortic valve replacement $(n=17), 196$ patients were enrolled in this study.

This study was approved by the institutional ethics committee/review board of Asan Medical Center, which waived the requirement for informed patient consent because of the retrospective nature of this study.

\section{Preoperative Evaluations}

CT scans with contrast enhancement were preoperatively performed on all patients, using a variety of scanners. Axial images of the branching vessels from the aortic arch to below the iliac bifurcation were obtained in contiguous 2.5- to 10-mm-thick sections. The maximal diameters of the aortic roots were measured from the initial images.

Two-dimensional echocardiography and Doppler color-flow imaging were performed on all patients before their operations using a HewlettPackard Sonos 2500 or 5500 imaging system equipped with a $2.5-\mathrm{MHz}$ transducer (Hewlett-Packard, Andover, Mass). In patients who presented with severe hemodynamic instability, the CT scan was performed as the first diagnostic modality and intraoperative transesophageal echocardiography was conducted during the anesthetic induction and surgical opening in the operating room to evaluate valvular lesion and left ventricular function.

\section{Surgical Procedures}

A standard median sternotomy was performed on all patients. Total cardiopulmonary bypass was instituted by arterial cannulation of the axillary artery $(\mathrm{n}=83)$, the femoral artery $(\mathrm{n}=58)$, both axillary and femoral arteries $(\mathrm{n}=52)$, or the distal ascending aorta $(\mathrm{n}=3)$. The timing and sites of cannulation were decided individually, according to the attending surgeon's preference and patient's status. The distal ascending aorta was selected as an arterial cannulation site among patients who underwent ascending aortic replacement for $\mathrm{AD}$ limited to the ascending aorta (DeBakey type II AD). During circulatory arrest, selective antegrade cerebral perfusion $(n=71)$, nonselective antegrade cerebral perfusion $(n=4)$, retrograde cerebral perfusion $(n=107)$, or both antegrade and retrograde cerebral perfusion $(\mathrm{n}=2)$ were used for cerebral protection, and moderate $(\mathrm{n}=57)$ (esophageal temperature $\left.\geq 20^{\circ} \mathrm{C}\right)$ or deep hypothermia $(\mathrm{n}=139)$ (esophageal temperature $<20^{\circ} \mathrm{C}$ ) was introduced according to the attending surgeon's preference.

Conservative root surgery was defined as preservation of the aortic sinus and valve. The proximal end of the aortic replacement was placed at the sinotubular junction in all patients using various methods to resuspend the sinotubular junction (ring in 64 patients, graft segment in 1 patient, Teflon felt strip in 66 patients, bovine pericardial strip augmentation in 1 patient, or pledgeted horizontal sutures in 64 patients). The entire tubular portion of the ascending aorta was resected. In conjunction with ascending aortic replacement, total arch replacement and hemiarch replacement were performed in 41 patients (20.9\%) and 152 patients (77.6\%), respectively; and ascending aortic replacement alone was performed in 3 patients $(1.5 \%)$ according to the individual patient's condition, intimal tearing site, or diameter of the distal arch. Concomitant coronary artery bypass was performed in 13 patients $(6.6 \%)$.

\section{Follow-up}

Data were obtained from each patient's medical chart during regular visits to the outpatient clinic or by telephone contact. Data on each patient's vital status were acquired from the Korean National Registry of vital statistics. Operative mortality was defined as death within 30 days after surgery.

The survivors at 6 months postsurgery received follow-up using transthoracic echocardiography or CT. The AR grades and maximal diameters of the aortic roots were obtained at the last follow-up echocardiograms and CT scans, respectively.

\section{Statistical Analysis}

Categoric variables and continuous variables are presented as numbers and percentages and as mean \pm standard deviation, respectively. The actuarial survival curve and event-free probability were calculated using the Kaplan-Meier method. The AR grades were compared between the preoperative echocardiograms and the last follow-up echocardiograms using the paired $t$ test. To determine the risk factors of the composite of significant AR (greater than AR grade 2+) and the aortic root dilatation $(>45 \mathrm{~mm}$ ), the Cox proportional-hazards model was used. The predictive value of the initial aortic root diameter for the composite of significant AR and root dilatation after aortic root conservative repair was evaluated using the area under the receiver operating characteristic curve, with $95 \%$ confidence intervals (CIs). The optimal cutoff corresponded to the value with the greatest accuracy (sensitivity + specificity). Event-free probabilities according to the cutoff value were obtained using the Kaplan-Meier method and compared using the log-rank test. Statistical significance is denoted by $P$ values $<.05$. The Statistical Package for the Social Sciences (version 18.0; SPSS Inc, Chicago, Ill) was used to perform the statistical analyses.

\section{RESULTS \\ Baseline Characteristics and Operative Outcomes}

The patients' baseline characteristics are presented in Table 1 . The 30 -day mortality rate was $5.1 \%$ (10 patients) and in-hospital mortality rate was $8.2 \%$ (16 patients). During a median follow-up of $45.3 \pm 36.4$ months (range, 0.06-130.0 months), there were 28 late deaths (14.4\%) and 1 case of proximal aortic reoperation $(0.5 \%)$. This patient, whose case had been diagnosed with Loeys-Dietz syndrome, underwent a reoperative Bentall operation in conjunction with replacement of the total aortic arch because of an extension of the preexisting AD that manifested 2 years after replacement of the ascending aorta and hemiarch for acute DeBakey type II AD. There were 10 cases of distal aortic reoperation $(5.1 \%)$, including descending thoracic aortic replacement and thoracoabdominal aortic replacement. Another 12 patients $(6.2 \%)$ underwent surgery for peripheral malperfusion, which consisted of femoral-to-femoral bypass and axillo-femoral bypass surgery. Overall survival and aortic reoperation-free survival at 5 years were $81.3 \% \pm 3.2 \%$ and $92.9 \% \pm 2.5 \%$, respectively (Figure 1). 
TABLE 1. Baseline characteristics of patients

\begin{tabular}{lc}
\hline \multicolumn{1}{c}{ Parameters } \\
\hline Total no. of patients \\
Demographic and baseline risks & 196 \\
$\quad$ Age, y & $56.9 \pm 11.4$ \\
Female gender, n (\%) & $100(51.0)$ \\
Marfan or Loeys-Dietz syndrome, n (\%) & $6(3.1)$ \\
Type of AD, n (\%) & \\
DeBakey type I & $152(77.6)$ \\
DeBakey type II & $17(8.7)$ \\
DeBakey subtype III-D (retro-A) & $27(13.8)$ \\
Intramural hematoma, n (\%) & $9(4.6)$ \\
AR grade, n (\%) & \\
None & $71(36.2)$ \\
1+ & $62(31.6)$ \\
$2+$ & $25(12.8)$ \\
$3+$ & $19(9.7)$ \\
$4+$ & $19(9.7)$ \\
\hline$A D$, Aortic dissection; $A R$, aortic regurgitation.
\end{tabular}

\section{Risk Factors for the Development of the Composite of Significant Aortic Regurgitation and Aortic Root Dilatation}

Among the 177 patients who survived 6 months or more after surgery, echocardiography and CT evaluations during the late period ( $>6$ months) were possible in $115(65.0 \%)$ and $138(78.0 \%)$, respectively (mean follow-ups, $44.9 \pm$ 32.7 months and $41.8 \pm 31.3$ months, respectively). During these periods, significant AR (greater than grade 2+) was observed in 5 patients $(4.3 \%)$, including 3 patients $(2.7 \%)$ without aortic root dilatation $(>45 \mathrm{~mm})$, and aortic root dilatation was observed in 19 patients $(13.8 \%)$, including 14 patients $(10.1 \%)$ without significant AR. The 3-, 5-, and 7-year freedom rates from the composite of significant AR and aortic root dilatation were $86.1 \% \pm 3.6 \%, 84.6 \%$ $\pm 3.9 \%$, and $80.2 \% \pm 5.7 \%$, respectively (Figure 2 ).
On the Cox regression analysis, the maximal aortic root diameter at the initial presentation was the only significant predictor for the composite of significant $\mathrm{AR}$ and aortic root dilatation (hazard ratio, $1.10 ; 95 \% \mathrm{CI}, 1.02-1.19 ; P=.014$ ).

When the predictive value of the initial aortic root diameter for significant AR and aortic root dilatation was estimated using the receiver operation characteristic curve, the curve yielded an area under the curve of $0.71(95 \%$ CI, $0.56-0.87 ; P=.011)$. The greatest accuracy for the prediction of significant AR and aortic root dilatation was obtained using a cutoff value of $47.5 \mathrm{~mm}$, which yielded $75 \%$ sensitivity and $58.7 \%$ specificity (Figure $3, A$ ). When freedom from significant AR and aortic root dilatation was assessed according to the cutoff value of $47 \mathrm{~mm}$, significant AR and aortic root dilatation developed earlier in patients with an initial aortic root diameter more than $47 \mathrm{~mm}$ compared with those with an initial aortic root diameter of 47 $\mathrm{mm}$ or less (Figure $3, B$ ). At the end of the follow-up period, the AR grades were significantly improved in comparison with the preoperative evaluations $(P<.001$, Figure 4$)$.

\section{DISCUSSION}

Acute type A AD is the most serious cardiovascular disease that requires an emergency operation. It is associated with high mortality and morbidity, which have remained considerable over time. ${ }^{5-8}$ Even in recent international studies, the early mortality of surgically treated patients with acute type $\mathrm{A} A \mathrm{AD}$ has been reported to be as high as $10 \%$ to $25 \%{ }^{9-11}$ In view of the high operative mortality, surviving the acute event is obviously of critical importance.

There is controversy regarding which surgical technique to use to repair the aortic root at the time of the initial operation for acute type A AD. Some authors have reported that the early mortality of acute type A AD remains significant, but this is not ordinarily influenced by the magnitude of the
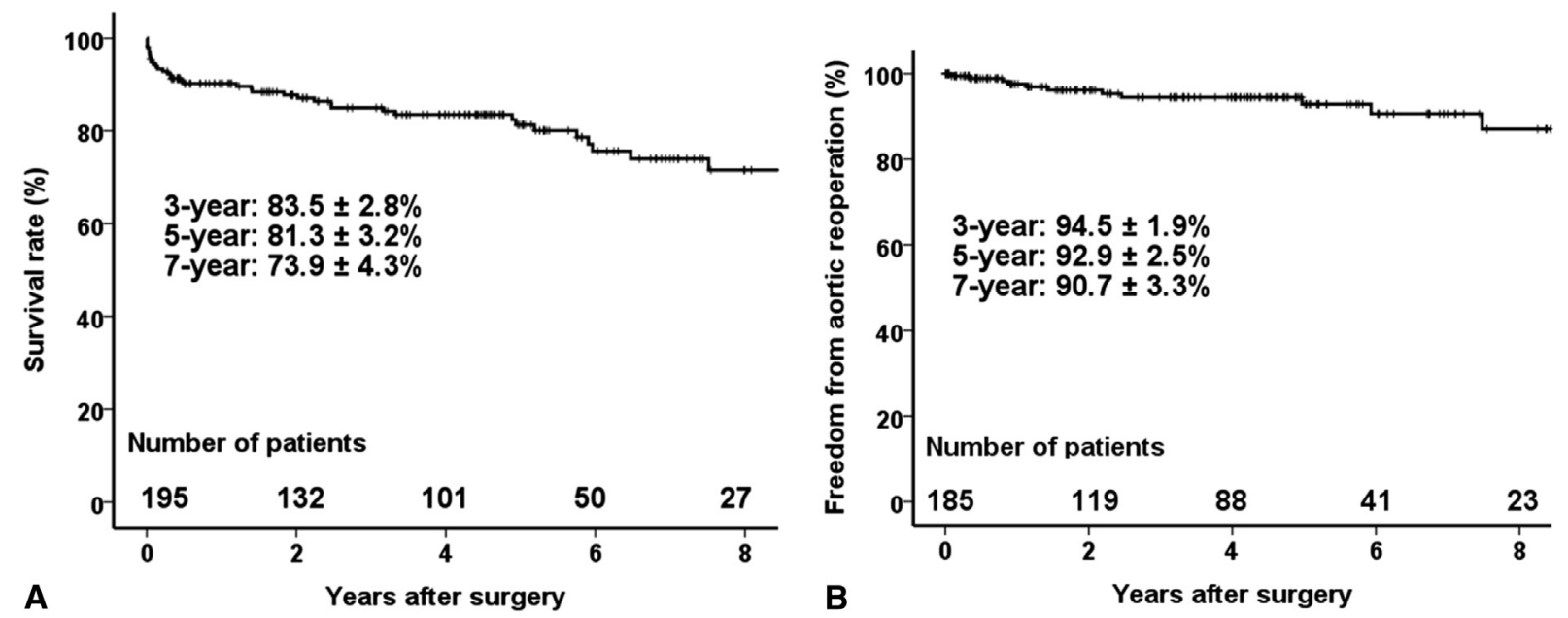

FIGURE 1. A, Actuarial survival curve of all patients. B, Freedom rate curve of proximal and distal aortic reoperation. 
surgical technique. ${ }^{4}$ Montalvo and colleagues ${ }^{4}$ reviewed 154 consecutive patients who underwent surgical repair of acute type $\mathrm{A} \mathrm{AD}$ and compared patients who underwent root reconstruction or repair $(n=44)$ with those who did not $(\mathrm{n}=110)$. There was no significant difference in the overall hospital mortality between these 2 groups. They suggested that preoperative shock, postoperative ventricular arrhythmias, and packed red blood cell transfusion are the risk factors for hospital death, rather than the extent of the operative procedure. Concistre and colleagues ${ }^{12}$ analyzed 250 patients who underwent emergency surgery for acute type $\mathrm{A} A D$ to determine the risk factors for reoperation after the initial surgery. They reported that nonreplacement of the aortic root is a significant and independent risk factor for proximal reoperation (relative risk, $2.14 ; 95 \% \mathrm{CI}$, 1.06$1.56 ; P=.0004$ ), although a more extensive procedure that includes both aortic root replacement and arch replacement increases operative mortality. In patients with connective tissue disease such as Marfan syndrome or Loeys-Dietz syndrome, sufficient resection of diseased aortic tissue via extensive aortic root surgery may prevent the necessity of a reoperation because of aortic root dilatation or the progression of AR. In our study, a patient with Loeys-Dietz syndrome underwent a reoperative Bentall operation in conjunction with replacement of the total aortic arch 2 years after replacement of the ascending aorta and hemiarch.

Some authors recommend the reimplantation technique that was first described by David and Feindel ${ }^{13}$ as a surgical option for patients with acute type A AD. ${ }^{3}$ They analyzed 295 patients who underwent supracommissural replacement of the ascending aorta $(\mathrm{n}=145)$, composite aortic valve and aortic root replacement $(n=64)$, and aortic valve-sparing reimplantation $(n=48)$. They reported that the reimplantation technique demonstrated clinical and functional results comparable to the established techniques

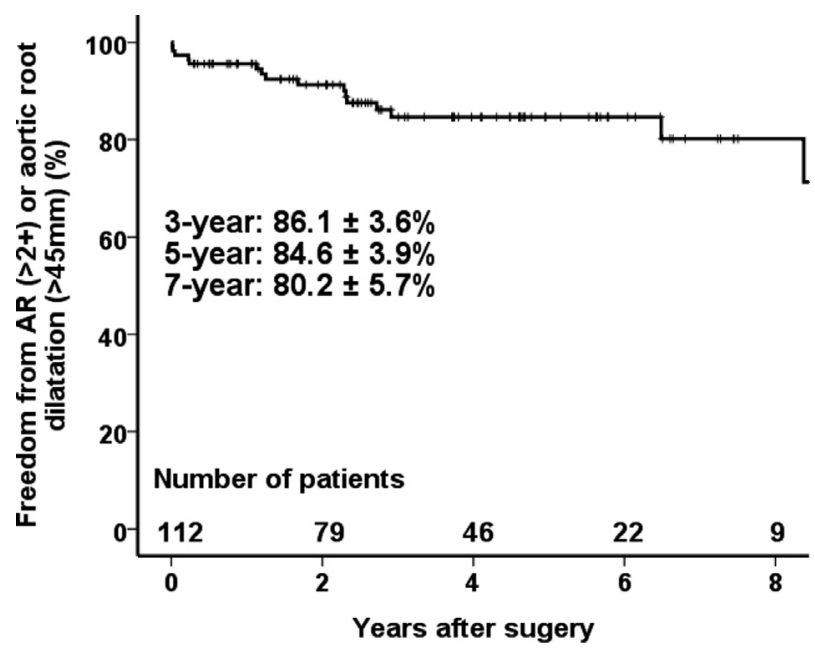

FIGURE 2. Freedom rate curve of the composite of significant $A R$ (greater than grade 2+) and aortic root dilatation (maximal aortic root diameter $>45 \mathrm{~mm})$. $A R$, Aortic regurgitation.

with a trend toward lower early mortality compared with supracommissural replacement and composite replacement despite the longer operation times. Theoretically, the aortic valve-sparing reimplantation technique is an appropriate surgical procedure for patients who present with acute type $\mathrm{A} \mathrm{AD}$ and morphologically normal valve cusps, because it allows the complete removal of diseased tissue and excellent hemostasis, and avoids the use of lifelong anticoagulation. However, it is not easy for inexperienced surgeons to precisely and safely perform this technique during emergency situations because it is a demanding technique that could prolong the operation time.

On the other hand, some authors have emphasized the benefits of conservative treatment for managing the aortic root in patients with acute type $\mathrm{A}$ AD. ${ }^{1,2,14,15}$
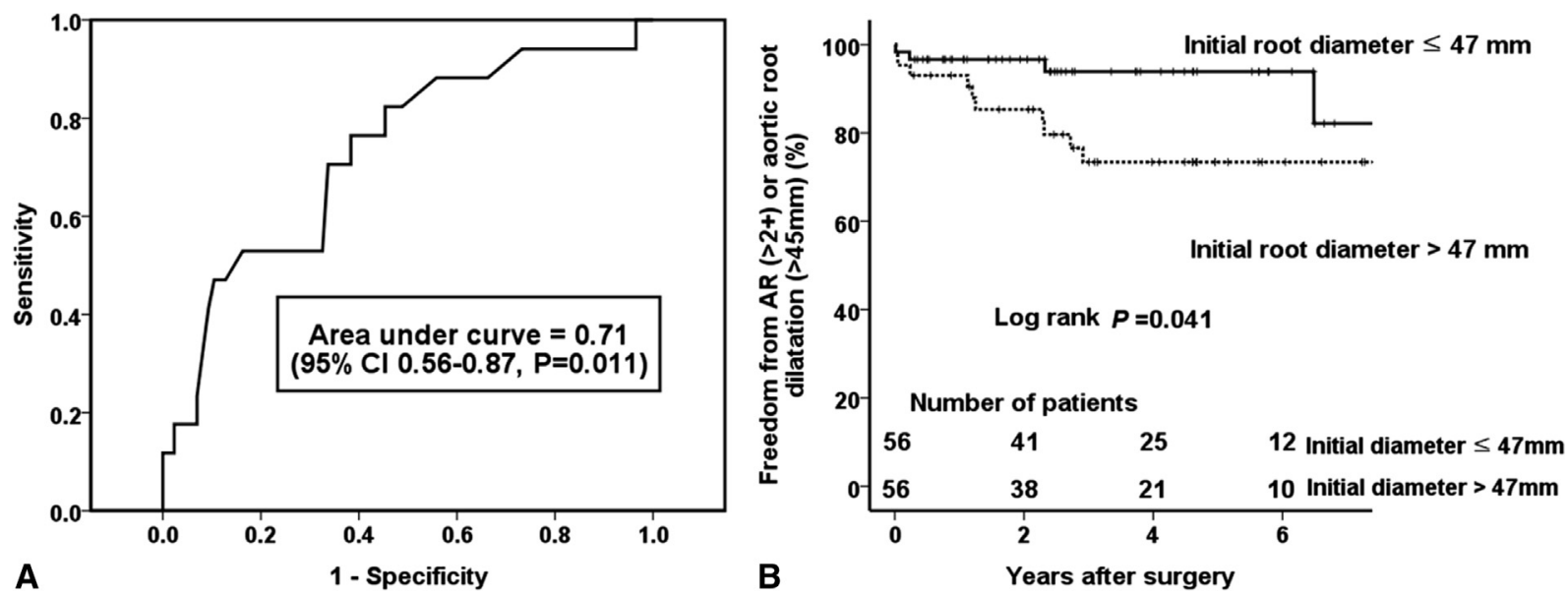

FIGURE 3. A, Receiver operating characteristic curve used to obtain cutoff values of the maximal aortic root diameter associated with the composite of significant AR and aortic root dilatation. B, Freedom rate curve of the composite of significant AR and aortic root dilation according to a cutoff value of 47 $\mathrm{mm}$. AR, Aortic regurgitation; $C I$, confidence interval. 


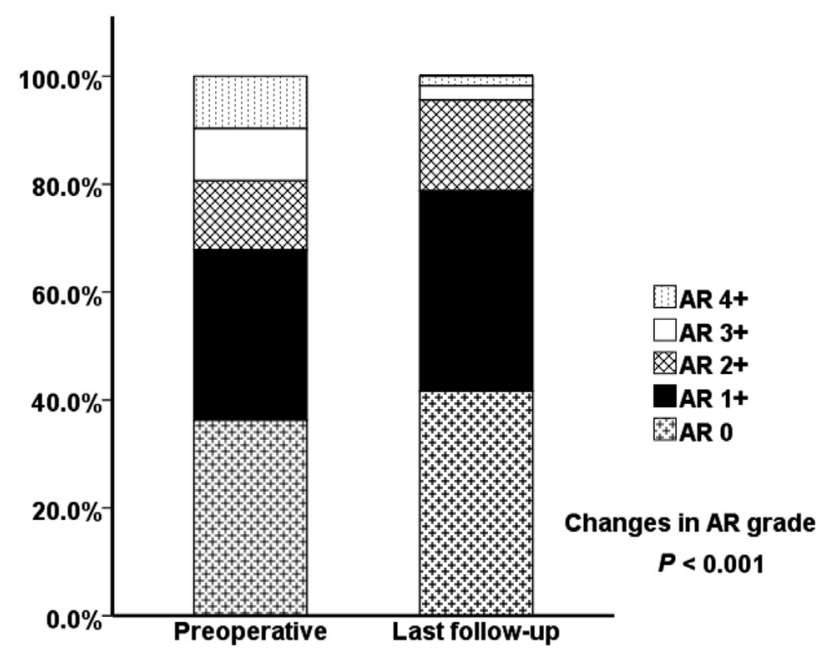

FIGURE 4. Comparison of AR grades between preoperative and last follow-up echocardiograms. $A R$, Aortic regurgitation.

Niederhauser and colleagues ${ }^{1}$ analyzed 199 patients who underwent surgery for acute type A AD and concluded that preservation of the aortic root is safe in the absence of Marfan or annuloaortic ectasia. Lai and colleagues ${ }^{15} \mathrm{di}-$ vided 123 patients into the composite valve graft replacement group, the separate valve and graft replacement group, and the commissural resuspension group and compared the overall survival and reoperation rates of these groups. There were no significant between-group differences. Westaby and colleagues ${ }^{2}$ introduced their policy of primary tear excision and preservation of the native aortic valve for the treatment of acute type A AD. They reported relatively low 30 -day mortality $(5.3 \%)$, which may be associated with their policy as they asserted.

Mazzucotelli and colleagues ${ }^{14}$ analyzed the long-term echocardiographic and clinical outcomes after preservation of the aortic valve for the treatment of acute type A AD. They concluded that preservation of the aortic valve during surgery for acute type A AD may be a valuable choice whenever feasible regardless of the severity of AR. In our study, the degree of AR significantly improved after conservative aortic root surgery $(P<.001)$. In addition, the relatively lower early mortality rate $(5.1 \%)$ might be attributed to this conservative approach. To obtain additional data, we tried to include postoperative CT data in this analyses in an effort to evaluate the aortic root more comprehensively, and we determined that relevant aortic root dilatation (maximal aortic root diameter $>45 \mathrm{~mm}$ ) occurred in $9.7 \%$ of patients and that the maximal root diameter at initial presentation was the only significant predictor of the composite of AR and root dilatation. In an effort to verify the consistency of the study results, we tried to compare the results of the present study with other published data. Because of the paucity of relevant data in the literature, however, no study was identified that examined the association between preoperative variables and postoperative aortic root/valve pathology in the setting of conservative root surgery for acute AD.

In the present study, the whole luminal diameter of the aortic root at the time of $\mathrm{AD}$ was predictive of composite adverse outcomes of root dilatation and aortic insufficiency. In patients with initial root dilatation, however, some may have preserved true lumen size, but only a dilated false lumen with thrombosis. These patients may exhibit different prognosis compared with those with true lumen expansion, but this hypothesis could not be tested in this study. This is attributable to the difficulty in measuring the false and true lumina at the level of the aortic root because of its 3dimensional shape; therefore, we could not incorporate such data in the multivariable model in this study. At the same time, we expect that patients with the dilated whole lumen, regardless of the size of the true lumen, may have a higher risk for reexpansion of the aortic root, referring to our previous report that dilatation of the whole lumen, neither false nor true lumen, was the only significant predictor for late development of descending aortic dilatation after aortic surgery for acute DeBakey type I AD. ${ }^{16}$

Another noticeable observation in our study is that not all patients with aortic dilatation (13.8\%) presented with significant AR $(4.3 \%)$. Likewise, only $22.8 \%$ of patients with aortic root aneurysm presented with severe AR in the report by David and colleagues. ${ }^{17}$ According to a study by Furukawa and colleagues, ${ }^{18}$ AR was not caused by dilatation of the aortic sinuses, but by dilatation of the sinotubular junction, aortic annulus, or both. This may explain why significant AR is not necessarily accompanied with ongoing aortic root dilation. Also, this would support our results that conservative root repair by resuspension of the sinotubular junction is a reasonable procedure for AR caused by acute type A AD.

In view of the complexity of aortic root replacement and the emergency characteristics of $\mathrm{AD}$, conservative repair of the aortic root for acute type $\mathrm{A} A \mathrm{AD}$ involving the aortic root may be a viable surgical option unless the patient has connective tissue disease or aortic root dilatation was observed on the initial examination. A larger number of patients is needed to obtain a universal value for the initial aortic root diameter that can be used to determine the extent of aortic root surgery needed to repair acute type A AD.

\section{Study Limitations}

The current study has some limitations. First, this study was limited by its retrospective nature. Second, comparison between conservative aortic root repair and aortic root replacement could not be performed because a control group was absent. Third, the risk factors for proximal aortic reoperation could not be obtained because proximal reoperation was performed in only 1 case. Instead, we obtained the risk factors for the composite of significant AR and aortic root 
dilatation. Finally, of the patients who survived for at least 6 months, $22 \%$ and $35 \%$ could not be evaluated by CT and echocardiography, respectively, during the late period, and therefore they were not included in the analysis. The significant dropout during the follow-up period may have affected the results of the current study.

\section{CONCLUSIONS}

Conservative aortic root repair of acute type A AD demonstrates acceptable operative mortality and long-term clinical outcomes. However, more aggressive approaches should be considered for patients with aortic root dilatation because of the risk of developing root aneurysms after surgery.

\section{References}

1. Niederhauser U, Kunzli A, Seifert B, Schmidli J, Lachat M, Zund G, et al. Conservative treatment of the aortic root in acute type A dissection. Eur J Cardiothorac Surg. 1999;15:557-63.

2. Westaby S, Saito S, Katsumata T. Acute type A dissection: conservative methods provide consistently low mortality. Ann Thorac Surg. 2002;73:707-13.

3. Kallenbach K, Oelze T, Salcher R, Hagl C, Karck M, Leyh RG, et al. Evolving strategies for treatment of acute aortic dissection type A. Circulation. 2004;110: II243-9.

4. Montalvo J, Razzouk A, Wang N, Bansal R, Rasi A, Hasaniya N, et al. Aortic root surgery does not increase the operative risk of acute type A aortic dissection. Am Surg. 2011;77:88-92.

5. DeBakey ME, McCollum CH, Crawford ES, Morris GC Jr, Howell J, Noon GP, et al. Dissection and dissecting aneurysms of the aorta: twenty-year follow-up of five hundred twenty-seven patients treated surgically. Surgery. 1982;92:1118-34.

6. Miller DC, Mitchell RS, Oyer PE, Stinson EB, Jamieson SW, Shumway NE. Independent determinants of operative mortality for patients with aortic dissections. Circulation. 1984;70:I153-64.
7. Crawford ES, Svensson LG, Coselli JS, Safi HJ, Hess KR. Aortic dissection and dissecting aortic aneurysms. Ann Surg. 1988;208:254-73.

8. Fann JI, Smith JA, Miller DC, Mitchell RS, Moore KA, Grunkemeier G, et al. Surgical management of aortic dissection during a 30-year period. Circulation. 1995;92:II113-21.

9. Bavaria JE, Brinster DR, Gorman RC, Woo YJ, Gleason T, Pochettino A. Advances in the treatment of acute type A dissection: an integrated approach. Ann Thorac Surg. 2002;74:S1848-52; discussion S57-63.

10. Trimarchi S, Nienaber CA, Rampoldi V, Myrmel T, Suzuki T, Mehta RH, et al. Contemporary results of surgery in acute type A aortic dissection: The International Registry of Acute Aortic Dissection experience. J Thorac Cardiovasc Surg. 2005;129:112-22.

11. Aalberts JJ, Boonstra PW, van den Berg MP, Waterbolk TW. In-hospital mortality and three-year survival after repaired acute type A aortic dissection. Neth Heart J. 2009;17:226-31.

12. Concistre G, Casali G, Santaniello E, Montalto A, Fiorani B, Dell'Aquila A, et al. Reoperation after surgical correction of acute type A aortic dissection: risk factor analysis. Ann Thorac Surg. 2012;93:450-5.

13. David TE, Feindel CM. An aortic valve-sparing operation for patients with aortic incompetence and aneurysm of the ascending aorta. J Thorac Cardiovasc Surg. 1992;103:617-22.

14. Mazzucotelli JP, Deleuze PH, Baufreton C, Duval AM, Hillion ML, Loisance DY, et al. Preservation of the aortic valve in acute aortic dissection: long-term echocardiographic assessment and clinical outcome. Ann Thorac Surg. 1993;55:1513-7.

15. Lai DT, Miller DC, Mitchell RS, Oyer PE, Moore KA, Robbins RC, et al. Acute type A aortic dissection complicated by aortic regurgitation: composite valve graft versus separate valve graft versus conservative valve repair. J Thorac Cardiovasc Surg. 2003;126:1978-86.

16. Kim JB, Lee CH, Lee TY, Jung SH, Choo SJ, Lee JW, et al. Descending aortic aneurysmal changes following surgery for acute DeBakey type I aortic dissection. Eur J Cardiothorac Surg. 2012 May 4 [Epub ahead of print].

17. David TE, Maganti M, Armstrong S. Aortic root aneurysm: principles of repair and long-term follow-up. J Thorac Cardiovasc Surg. 2010;140:S14-9; discussion S45-51.

18. Furukawa K, Ohteki H, Cao ZL, Doi K, Narita Y, Minato N, et al. Does dilatation of the sinotubular junction cause aortic regurgitation? Ann Thorac Surg. 1999;68: 949-54. 